\title{
A Form-Finding Instrument for Reciprocal Structures
}

\section{Udo Thönnissen}

\begin{abstract}
In order to span longer distances than the length of the available timber beams the structural principle of mutually supporting beams was used in vernacular buildings and in studies by numerous architects and scientists. Although the generating rules are simple, the control of these structures has to date proven to be very complex because of the reciprocal dependency of the elements. The paper describes the development of a digital tool that shall facilitate the design of these structures and enhance the spectrum of architectural applications. The structural configuration of an assembly is constituted by means of a form-finding process, which is based on the individual members and its relations. Several parameters for these members enable the user to respond to contextual conditions such as plan form, height and number of supports, and to gradually refine the output. The report ends with the description of three applications in which several wooden materials and various forms of fabrication have been tested.
\end{abstract}

Keywords Reciprocal structure - Spatial structures - Structural morphology · Timber constructions · Form-finding · Morphogenesis ·

History of construction

\section{Introduction}

Historical roots

When the designer Graham Brown rediscovered the structural principle in 1987 he renamed it the 'Reciprocal Frame' (Brown 1989). In the various studies on this kind of spatial structure it has been termed differently: mutually supporting beams

\footnotetext{
U. Thönnissen $(\bowtie)$

D_ARCH ETH Zurich, HIL G57, Wolfgang-Pauli-Str. 15, 8093 Zürich, Switzerland

e-mail: thoennissen@arch.ethz.ch
} 
(Brown 1989), lever-beam structures (Bertin 2002), and mutually supported element systems (Rizzuto 2007). The term nexorades was introduced by Baverel (2000).

Although very few built testimonies are remaining it is assumed that the principle of reciprocity has been known for a very long time and that it has been used to bridge larger spans with short interlocking beams. History records the presence of similar reciprocal constructions in oriental and occidental cultures, but their evolution is not linear and they are mostly unrelated to each other.

An extensive overview of the studies of architects, scientists and mathematicians, and realised buildings and applications has been made by Popovic Larsen (1996, 2008, 2009) and Pugnale and Sassone (2014).

Fundamentals of the System

In a reciprocal structure a minimum number of three elements support each other in such a way that each member rests on another and/or on the ground, and at some intermediate point or points supports at least one other element. If this condition is fulfilled, then this elementary configuration forms a single unit creating a fan-like structure. Following the terms proposed by Douthe and Baverel (2009), this fan has a rightward or leftward orientation (Fig. 1). The number of $n$ beams generates a polygonal form in plan with $n$ angles. With these configurations spans can be covered that are greater than the maximum length of the elements.

Unlike a grid structure with pin-joints, the elements form an open node or engagement window. The 'engagement length' $a$ of a member defines the distance of two points of the member that are connected with two other members of the same fan and constitutes one of the principal form-defining parameters. The definition that the members support each other in a spatial configuration implies an eccentric overlap of the members. The eccentricity $e$ is therefore the distance between the axes of two connected elements. If $e>0$, the loading elements are in a higher position than the supporting beams, and a structure with inclined members is formed. In planar reciprocal structures all members are placed in one level, that means that $e=0$ (Fig. 2). The flat grillages designed by Sebastiano Serlio,
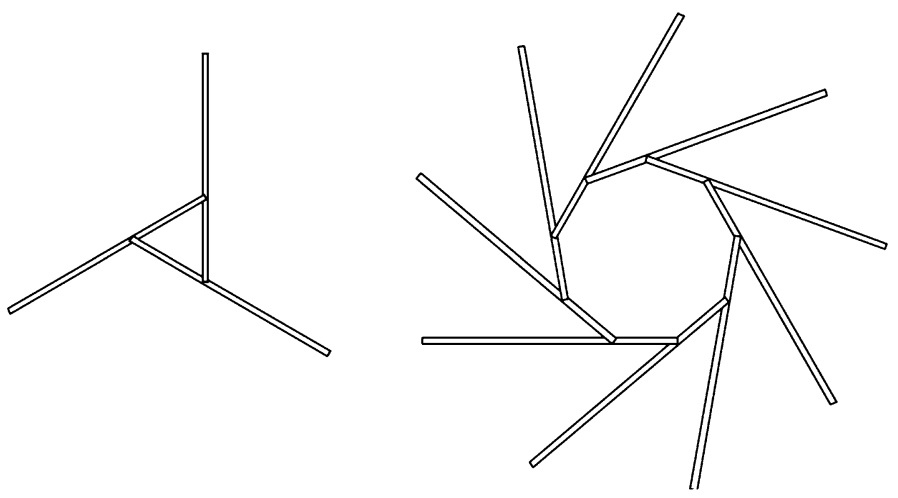

Fig. 1 Forms of reciprocal structures. Left rightward fan, right leftward fan 

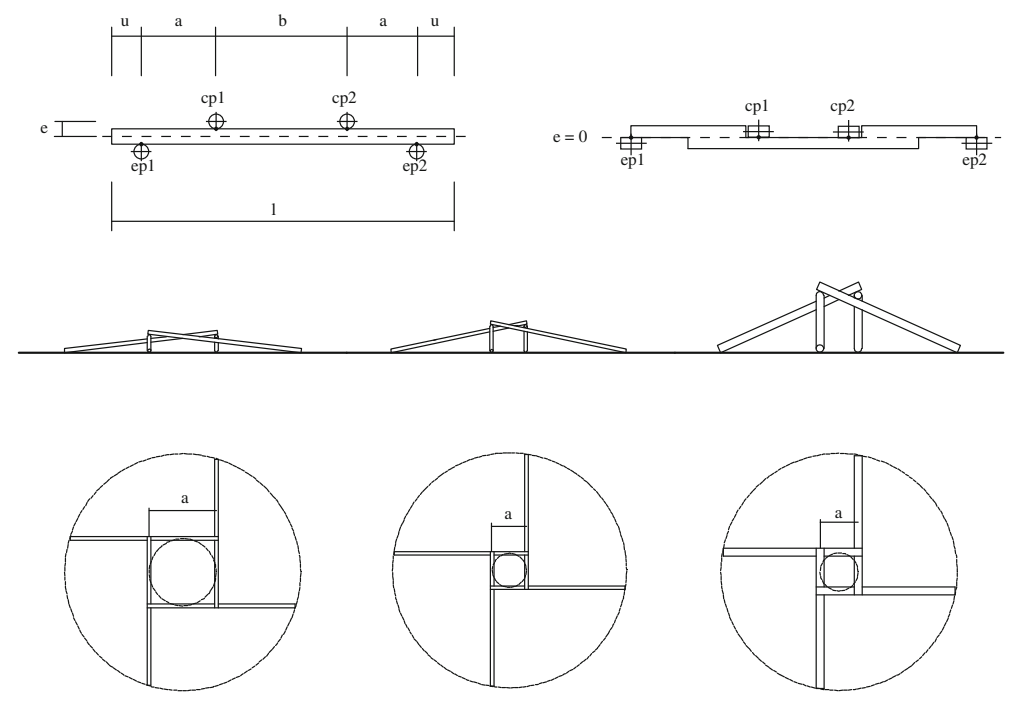

Fig. 2 Parameters $e, a$ and $l$ of reciprocal structures

Fig. 3 Multiple unit

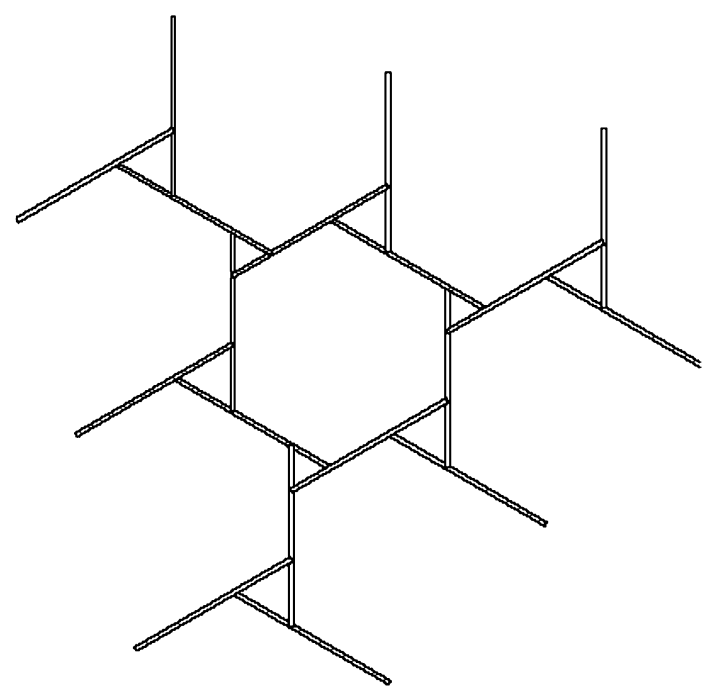

Leonardo da Vinci and John Wallis are examples of this type of structure (Houlsby 2014).

\section{From a Minimum Configuration to Patterns}

Composing several units in a way that each member is part of two units, different patterns will result (Fig. 3). Equal units can be added to infinite configurations (Bertin 2002). Since every member in the system, except the boundary elements, 

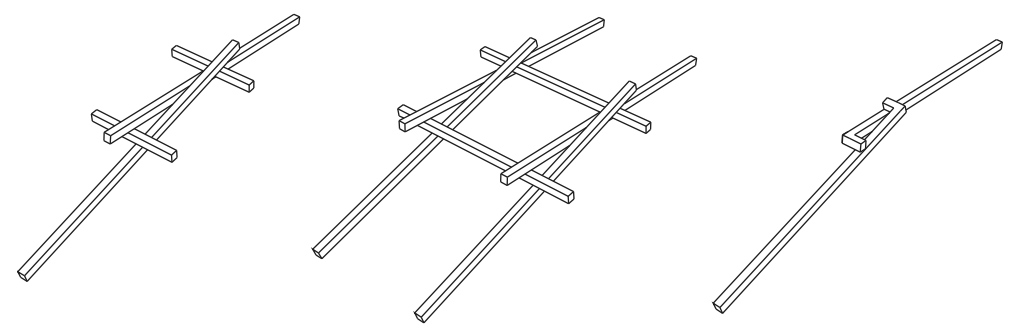

Fig. 4 Reciprocal structures with a parallel addition of linear elements

performs the same function within the whole, it can be regarded as a nonhierarchical structure.

The principle of reciprocity also is applicable with two members if they are connected with additive members such as coupled purlins (Fig. 4).

These coupled purlins enable the parallel addition of linear elements to a stable spatial configuration. If the members have the form of an $\mathrm{L}$ or an $\mathrm{S}$, then the levering in two directions is possible (Bertin 2002).

The global form of a reciprocal structure with elongated elements depends on a variety of parameters:

- style of the fans;

- number $n$ of members in the assembly;

- engagement length $a$ of a beam;

- eccentricity $e$ between the members;

- overall length $l$ of one member;

- configuration of members in the structure.

Since every element responds to the entire set of elements and to the associated parameters, any change in one value will influence the whole. Although reciprocal structures have been known to builders since time immemorial and can be regarded as a universal principle of construction, there has been only limited number of applications in architecture to the present, mostly single reciprocal frameworks. The reason might be the relatively flexible connections between the members, which prevent the constructions from resisting a long period of time. Above all, it is the complex behaviour of these structural frameworks that makes it extremely difficult for the designer and builder to anticipate how large assemblies react to changes in the configuration.

This present research is an attempt to control the complex morphogenesis in the design and to supply the necessary information for production and assembly. It shall thereby extend the spectrum of potential architectural applications and forms of expression.

\section{The Form-Finding Tool}

According to Pugnale and Sassone (2014) in reciprocal structures three different form-finding principles can be applied: 
(1) iterative additions;

(2) optimization strategies;

(3) modelling of kinematically undetermined systems

Here the focus is placed on the second principle, optimization strategy, which can follow two different approaches:

(A) The elements are orientated to a given surface. A built result of this method can be studied in the reciprocal network of the Rokko Observatory by Architect Hiroshi Sambuichi in collaboration with the Arup Group (Kidokoro and Goto 2011; Popovic Larsen 1996). Here a shift frame geometry developer formulated specifically for the project was applied.

In a further development the final geometrical and spatial configuration is achieved by means of the combination of gradient based algorithms and genetic algorithms (Parigi et al. 2012).

(B) Another approach starts from elementary configurations and their modification or deformation. These begin with planar or three-dimensional grids without eccentricity and engagement length as an initial configuration and use genetic algorithms or the dynamic relaxation method (Baverel et al. 2004; Douthe and Baverel 2009).

Diverging from the last approach, the process described in this paper starts with a planar configuration of cells that define the basic relations between the elements. The introduction of cells as initial configuration that drives the form-finding process allows local manipulations of the structure such as the insertion of openings, the displacement of bearing points in space or the variation of fan styles and patterns.

\section{Cell Relations}

The specification that a fan is an opened node with an arrangement of rotating members would suggest a subdivision of one unit into a cell and several interlocking elongated elements - denominated 'slats' — around it. As mentioned earlier, in an elementary configuration the minimum number of three members constitutes one cell with the radius $r$. Each slat can be perceived as a tangent to two cells, cell1 and cell2 (Fig. 5a). The index of each slat always contains two implicated cells with the corresponding angles to the $x$-axis. These angles serve as criteria to sort the slats in each cell (Fig. 5b).

\section{Extension}

For each cell the point TP1 is achieved by extending the tangent line slat[i].cell[t] until it meets the next slat in cell self. Extending P2 to the next member of the cell $n b r$ gives point TP2. During the iterations over all cells each cell is considered as cell self surrounded by neighbouring cells $n b r$ (Fig. 5c). 

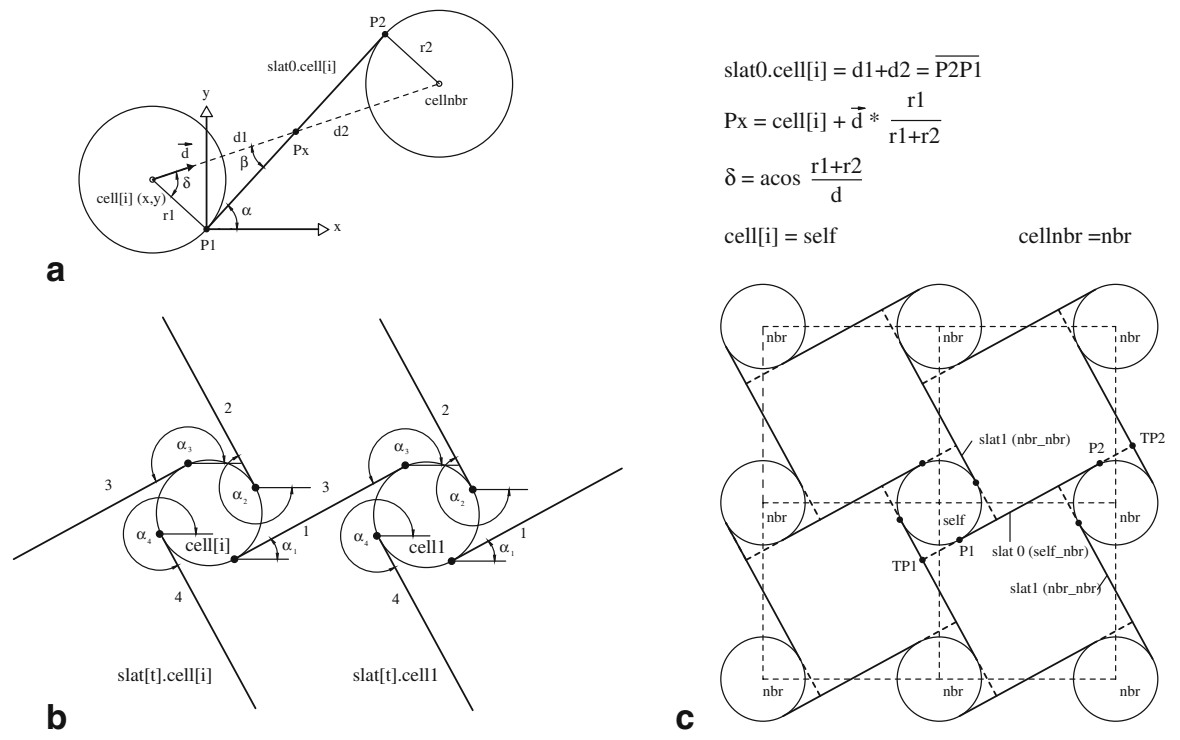

Fig. 5 Relation between two cells: a geometric relations; b angles of slats; $\mathbf{c}$ cell[i] and neighbouring cells

\section{Translation}

The same relation between the cell self and its neighbouring cells defines the translation. Once the tangents have been defined and extended, the respective points TP1 and TP2 are displaced on the member until the engagement length $a$ is reached, in this case at $l / 3$ (Fig. 6). They constitute the contact points CP1 and CP2 of the adjacent members.

A normal vector $e n$ of the plane $E$ indicates the direction of the eccentricity between two elements. As a precondition, all joints are exposed to friction and compressive force, therefore the value of the vector en is set positive by default.

The tangent points of the adjacent elements at the circle with the radius $e$ give the new contact points $\mathrm{CP} 1$ and $\mathrm{CP} 2$.

In a plan configuration the alignment along the longitudinal axis of each member is equal. In reciprocal structures with inclined members the alignment of the beams has to adapt to the inclination. Hence, the normal vectors $e 1$ and $e 2$ of the adjacent elements result in the vector sum $v n$ as the height vector of a member.

The steps translation and alignment are iterated in several loops over all cell indexes and its corresponding slats in the configuration. Depending on the number and the distribution of applied cells, after a minimum of thirty cycles the assembly converges to an optimal geometrical configuration (Fig. 7). The more irregular a cell distribution, the less congruence of the tangents in plan with the computed spatial relations is shown.

In order to automate the creation of the relational network between the cells the Delaunay triangulation is applied (Delaunay 1934). This function produces a 


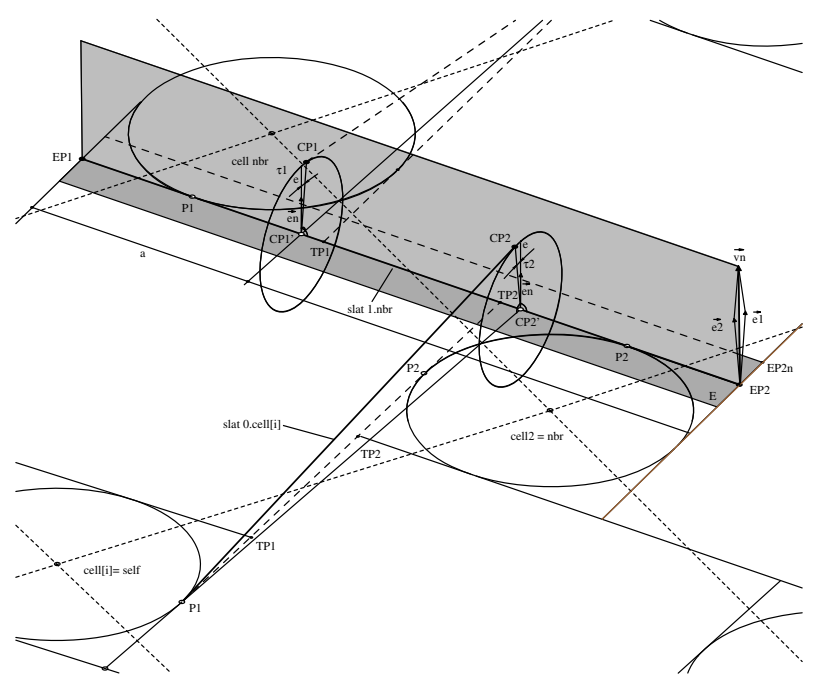

Fig. 6 Extension and translation of tangential lines

triangulated mesh out of a point cloud in which the edges of the triangles connect the nearest reachable points with each other (Fig. 8).

Provided that the fan style in each cell is equal, the contact elements meet a member from the opposite direction. If the fans rotate in such a way that one rightward fan is surrounded by leftward fans, the elements meet at the same side of the supporting member (Fig. 8). This has a crucial effect on the generation of patterns in the network.

The eccentricity $e$ and the engagement length $a$ are the most important parameters to control the global form. At this point the tool is limited to the work with symmetrical values $(a=c)$ to the benefit of an easier production and assembly (see also Fig. 12).

\section{Formation of the Members}

After the form-finding process for the global structure parameters related to the form of the members can be actuated.

Width of the Element $w$

The width $w$ determines the displacement of the longitudinal axis towards both sides in order to span a plane (Fig. 9).

Height of the Element $h$

The plane is extruded in both $z$-directions. The extrusion angle $\alpha$ is by default $90^{\circ}$ in order to achieve rectangular cross sections. 

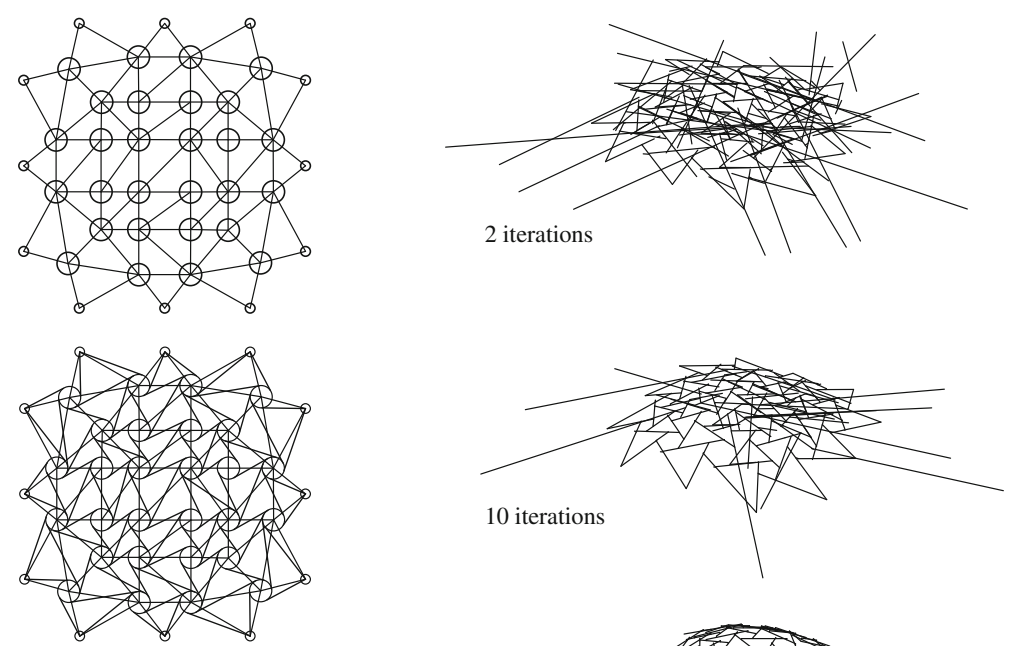

normal cell

- boundary cell

— Delaunay line

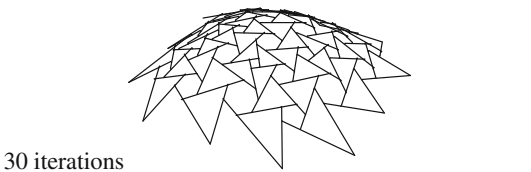

Fig. 7 Form-finding iterations
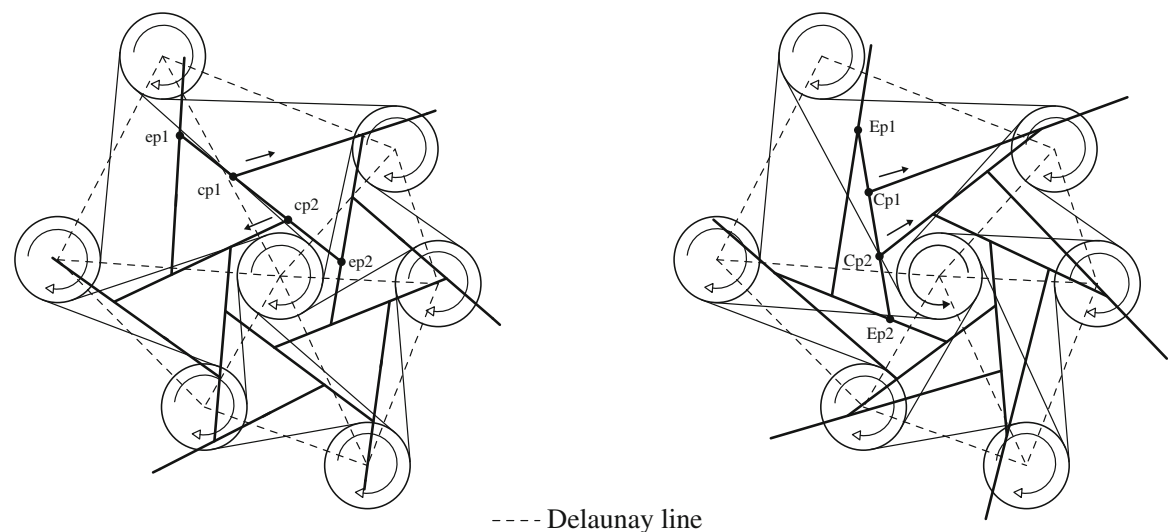

Fig. 8 Delaunay lines, leftward and rightward fans

Height and width determine whether a member has a beam-type or a plate-type form. The intersection of the members is important for the generation of the joints, which in the case of reciprocal structures should preferably be part of the members itself (Fig. 9).

\section{Excess Length $\mathrm{u}$}

For certain designs and for the generation of the joints the excess length $u$ at EP1 and EP2 is important (Fig. 10). 

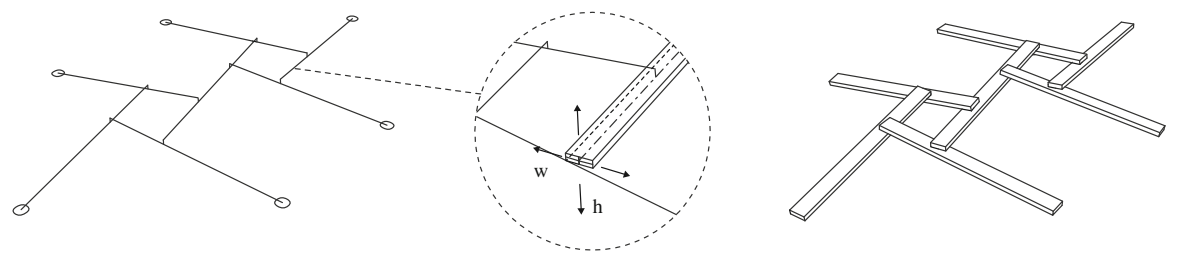

Fig. 9 Height $h$ and width $w$ of the members

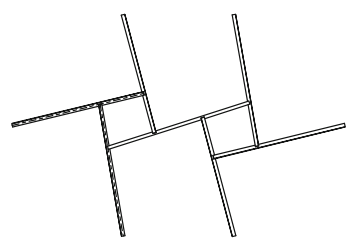

$\underbrace{\mathrm{a} \nabla \nabla^{\mathrm{c}}}$

$\mathrm{a}=\mathrm{c}=1 / 3$

$\mathrm{u}=0$

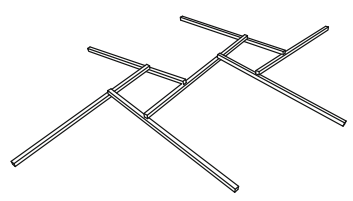

a
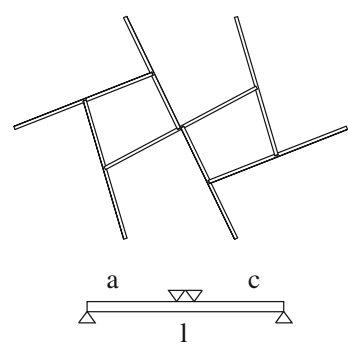

$$
\mathrm{a}=\mathrm{c}=0.49 * 1
$$$$
\mathrm{u}=0
$$

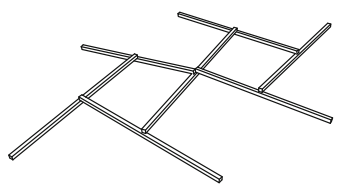

b
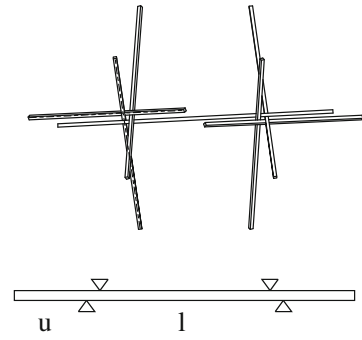

$\mathrm{a}=\mathrm{c}=0.05 * 1$

$\mathrm{u}=1 / 3$

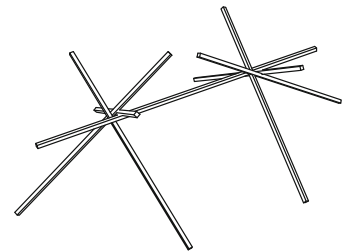

c

Fig. 10 Engagement length $e$ and excess length $u$

\section{Other Cell Types}

In order to enhance the scope for the designer and to respond to contextual circumstances, other cell types than the normal cells above shown are introduced. These follow other rules and have a specific influence on the form of the structure.

\section{Boundary Cells}

Boundary cells enable the designer to determine the members that are to act as supporting elements. Thus, the base points can be precisely placed in the centre of the circle that represents the boundary cell. The common problem of reciprocal structures with elongated members, not connected members on the edges of the framework, can be controlled in a better way. Delaunay lines do not connect the boundary cells to each other, i.e., here the structure ends with one, two or three members that touch the ground or the support. However, the boundary cells need not be placed only on the periphery, but can also generate a central 'strut' (Fig. 11). 

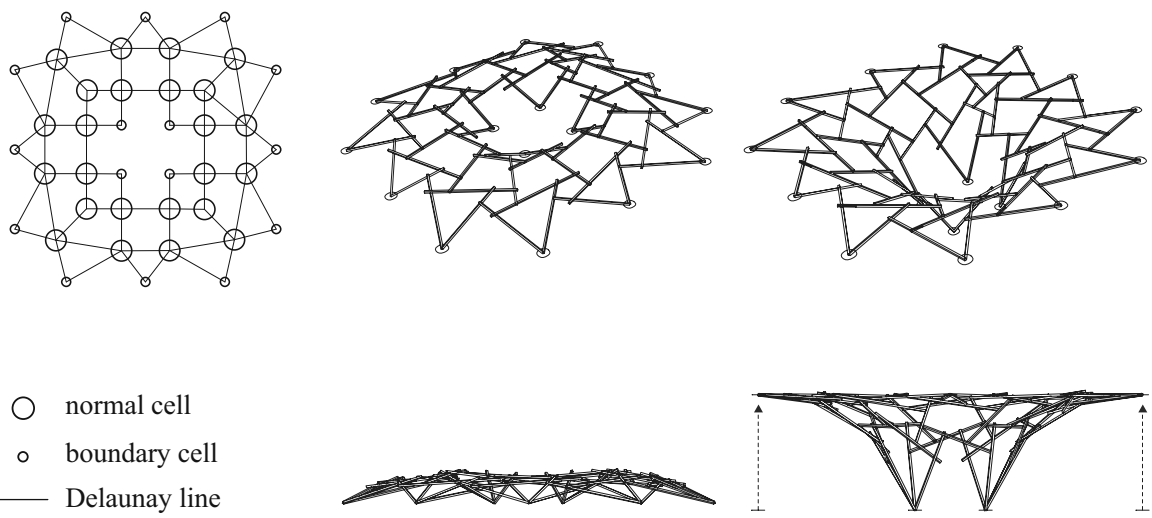

Fig. 11 Boundary cells

Moreover, the boundary cells can be shifted vertically to set up the structural framework on supporting points with different heights, or other structural elements, such as arches, can be used as supports. Likewise several reciprocal systems can be combined.

\section{Aperture Cells}

As shown in Fig. 4, for every normal cell the distribution of bearing members and support members along the beam follows the ratio $p n$ and can be changed by the user. In the case where the user wants to introduce an opening into the network, an aperture cell can be applied. In this cell the positions of the points CP1 and CP2 on the members change inversely. In this way the cell pattern can be expanded (Fig. 12). The engagement length of the aperture cell $p a=a / l$ can be modulated independently.

\section{Manipulations in the Drawing}

Once the user has drawn a setting in plan further modulations may be undertaken by adding respectively, erasing cells and Delaunay relations in the input.

\section{Manipulation of Delaunay Lines}

These manipulations enable the regulation of the number of members per cell, the connection of specific cells with each other, or the interruption of the triangular relationship between the cells (Fig. 13). This allows the development of varied patterns and the regulation of density. The directions of the lines have a crucial effect on the curvature of a framework. 

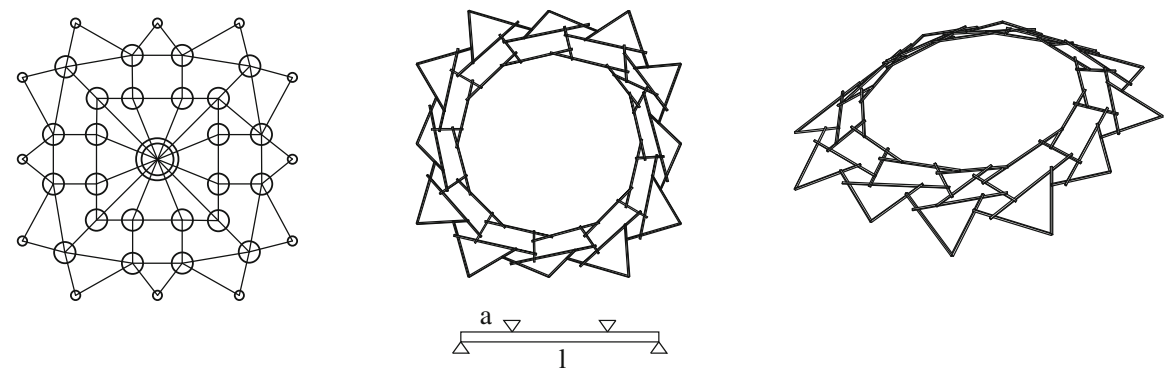

$\mathrm{pa}=\mathrm{a} / \mathrm{l}=\mathrm{l} / 4$
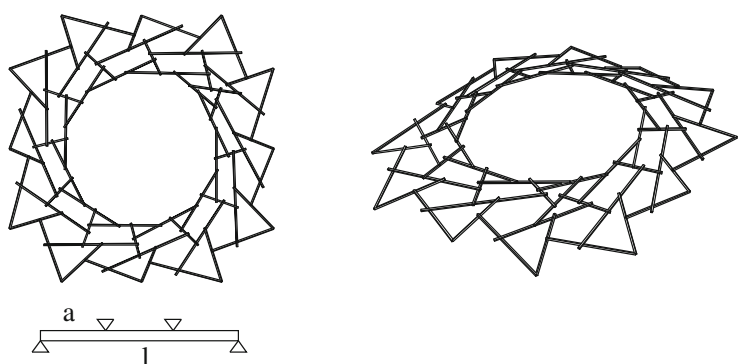

$\mathrm{pa}=\mathrm{a} / \mathrm{l}=1 / 3$

Fig. 12 Aperture cells
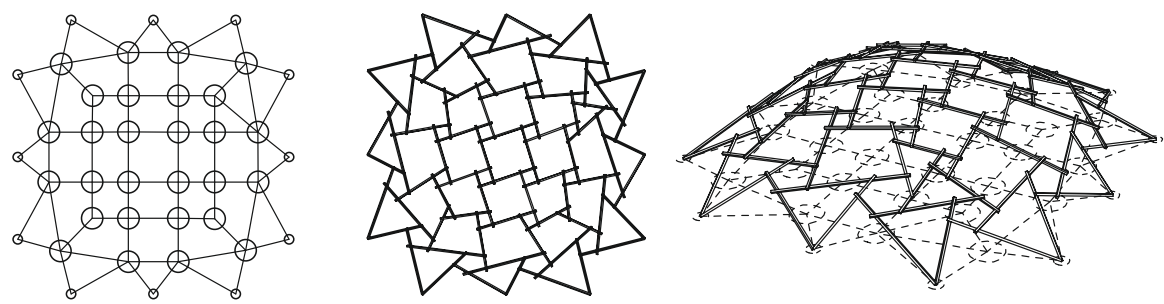

number of slats: 73
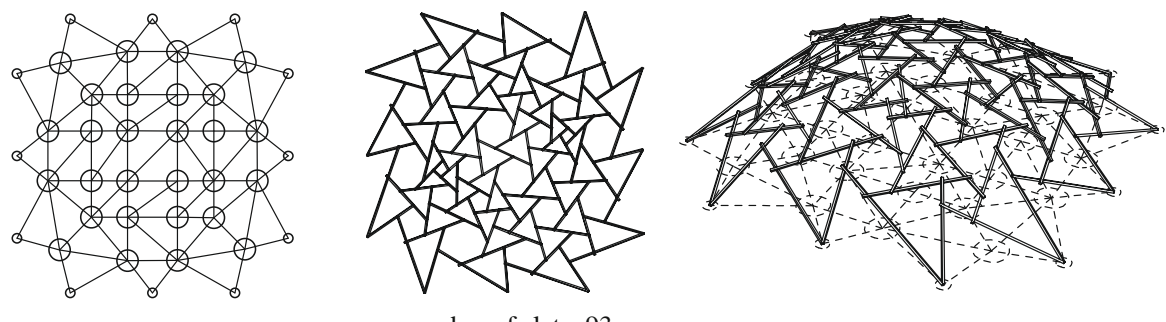

Fig. 13 Manipulations of Delaunay lines 

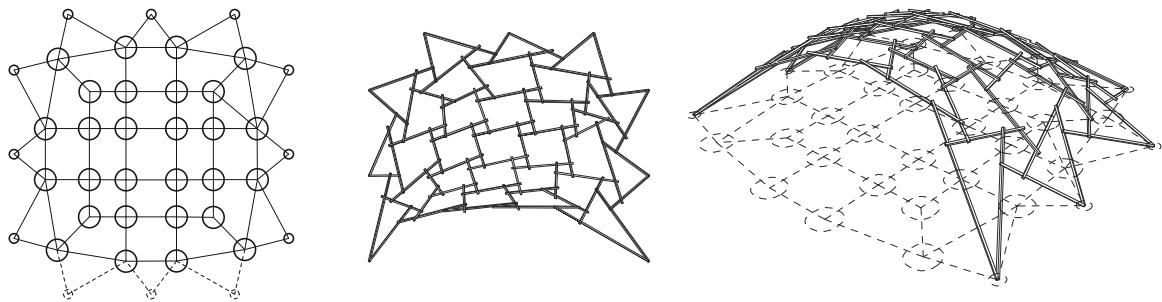

Fig. 14 Arches and openings

Arches

If several boundary cells and their connecting Delaunay lines are deleted, the members between the remaining boundary cells form an arch or an opening. The members are displaced in the $y$-and $z$-directions, until the initial condition for every beam is fulfilled (Fig. 14). The overall structure is distorted, leading to acute angles between the members, in some cases complicating the assembly of certain structures.

Close to the boundary members the forces act directionally in line with the members, resulting in transverse bending in the next element. In the output as platelike elements or beams with asymmetrical cross sections, the tool enables two countermeasures in order to slightly reduce transverse bending:

- Pivoting of boundary members. As opposed to the alignment of the normal cells where the orientation depends on the adjacent members, the boundary members can be controlled independently. The elements (in this case plate-type elements) are orientated so that they meet in an intersecting line at their ends. By rotating the boundary cell relative to the ground plane this line can be controlled in two directions, and thus the alignment of the boundary members can also be controlled;

- Displacement of boundary cells. Another option to trigger the boundary elements is to define a displacement as a fixed property of a specific boundary cell. In the code this cell receives another name and the associated shift. Thus, boundary elements that meet the ground plane in flat angles can be erected in order to reduce transverse bending.

\section{Input and Output}

Work with the tool requires a three-dimensional CAD environment that allows the implementation of plugins; in this case rhinoceros was used. Drawing the cells and the Delaunay lines as input for the computation generates the geometry. It is above all this visualization that gives the user control over the system. A python script has been developed that can be activated as a separate command. After the computation and visualization the input can be modified and the configuration is computed anew and re-visualized. Subsequently it is possible to export the member and joint geometries for the production and the static calculation. The information needed for the assembly can be exported in a separate text file. 


\section{Fabrication and Assembly}

In this process for the further development of reciprocal structures the so-called digital chain is both the starting and the end point. In this way any number of different elements can be manufactured and more complex systems can be created. The programme developed must therefore be able to supply the data from the designed structure to the production process. Rhinoceros allows the export of geometrical information in any necessary format, but the data must nevertheless be post-processed in a CAM environment to provide the exact fabrication format.

In what follows, several built case studies will be shown in order to explain the possibilities regarding fabrication and assembly.

\section{Pergola HIL}

In this small pergola for the entrance courtyard of the architecture faculty of ETH Zurich a differentiated structure was created by using simple $4 \times 6 \mathrm{~cm}$ timber slats. Steel bolts were used in order to secure the position of the members to each other. Sufficiently dimensioned holes were drilled compensating the torsion of the beams to each other. Consequently at the beginning of the assembly the structure is very flexible gaining structural and formal stability with every added element. Eventually the different lengths of the elements and their precise position in the network generate the overall form (Fig. 15). It is therefore essential to provide the position of each element and all adjacent elements in a so-called assembly code.

The assembly code contains the index of each member and of all attached members. Here the absolute position of a beam is not required; it is rather the relative position in the network that is important for the assembly. In Fig. 14 the beams 109 and 26 support the beam 25. In the opposite direction the members 34 and 28 bear down on it. Beginning with one beam all corresponding members can be assembled, and a plan for the erection is not necessary. However, due to the

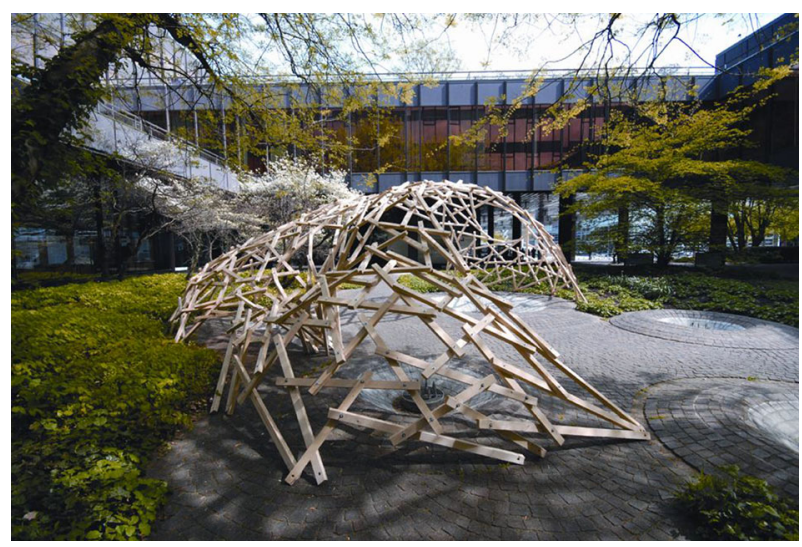

Fig. 15 Pergola HIL. Photo: courtesy Christopher Rofe 
symmetric position of the holes the framework might be mounted reversely. The boundary beams, for example beam 109, have reduced connection points, and are therefore marked with an $x$. Theoretically the erection can be started with any member, but in the case of complex frameworks it should begin with the most complicated parts, in this case the central 'column'.

The output also contains all the data for the fabrication, which in this case was undertaken manually using the simplest of tools. Each element has a specific length $l$ and a fixed symmetric engagement length $a=c$ (Fig. 16), facilitating the placement of the drill holes along the beam. The symmetric position of the holes also enables the easy integration into the network.

\section{Pergola SC}

This Pavilion was executed on the campus of ETH Science City (Fig. 17). In a different line of development the use of plate-like materials was tested. For each member a contour section with specific distances from the support points is computed and extruded according to the available material (Fig. 18).

Hence, the members contain more 'formative' information and the outline is not symmetrical anymore. Because of the smoother overall form eventual claddings can be added more easily. After the form-finding procedure all the elements are orientated in the ground plane, and the assembly code is written on the left side of each member. The assembly code in Fig. 18 contains the element number and the numbers of the four adjacent members.

With a nesting plug-in for rhinoceros, such as RhinoNest, the position and orientation of the elements can be optimized for a given panel size. Subsequently
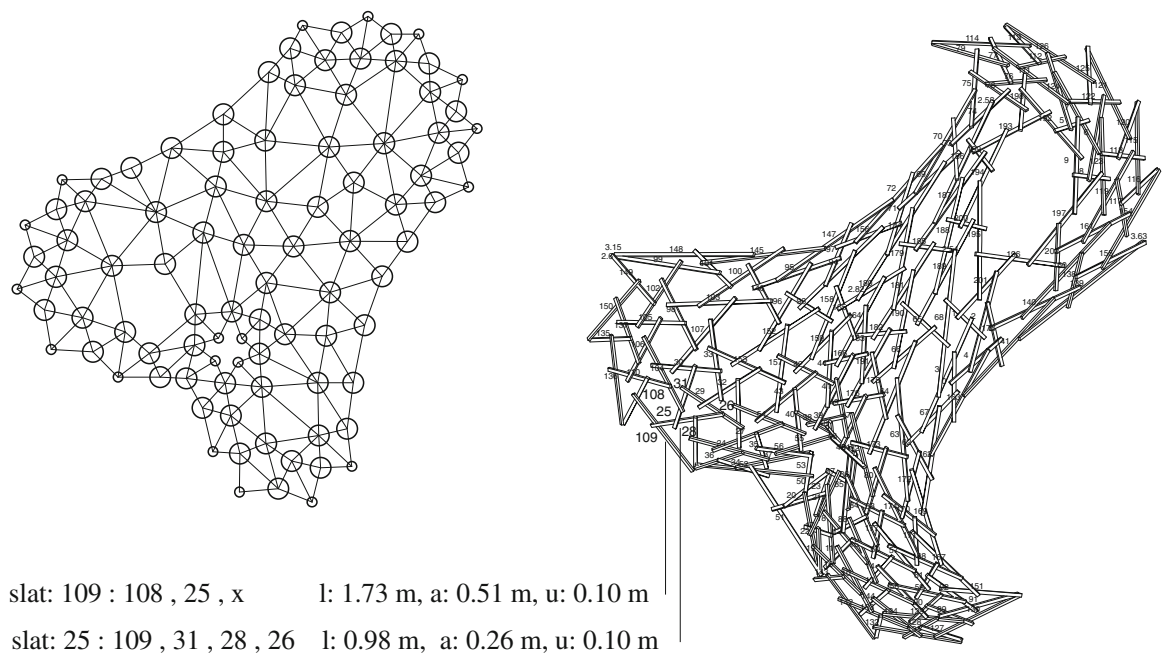

slat: $109: 108,25, \mathrm{x}$

1: $1.73 \mathrm{~m}, \mathrm{a}: 0.51 \mathrm{~m}, \mathrm{u}: 0.10 \mathrm{~m}$

slat: $25: 109,31,28,26$

l: $0.98 \mathrm{~m}$, a: $0.26 \mathrm{~m}, \mathrm{u}: 0.10 \mathrm{~m}$

Fabrication

Fig. 16 Design of HIL Pavilion with assembly and fabrication code 
Fig. 17 SC Pavilion. Photo: courtesy Peter Hauser
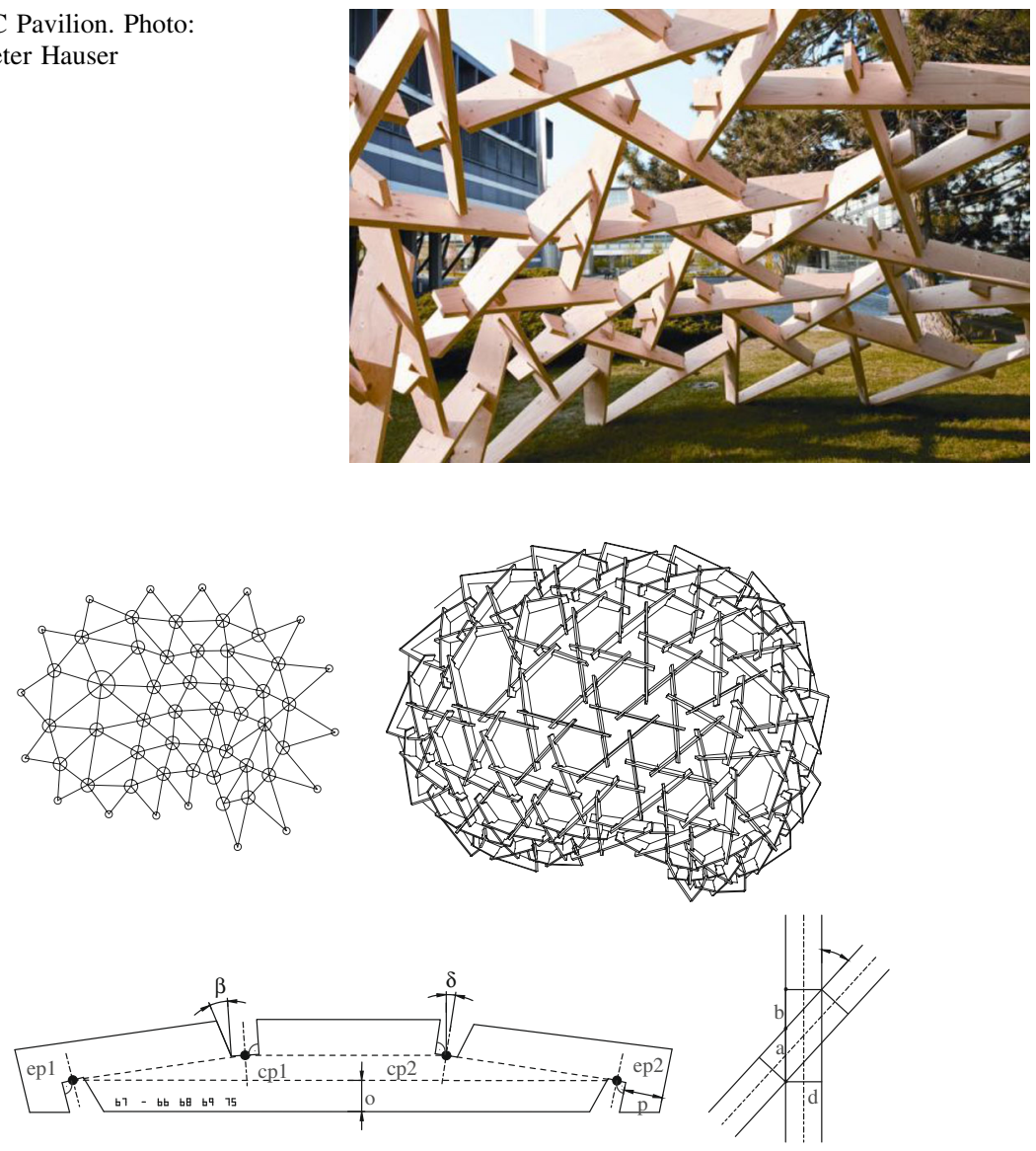

$\beta=$ angle of notch opening
$\delta=$ angle of adjacent members
$o, p=$ offset distances around connecting points ep1,ep2,cp1,cp2

$\mathrm{a}+\mathrm{b}=$ width of notch

$\tan (\alpha)=d / b$

$\sin (\alpha)=\mathrm{d} / \mathrm{a}$

$a+b=d / \sin (\alpha)+d / \tan (\alpha)$

Fig. 18 Design of SC Pavilion, notch geometry

the file is exported to a CAM-module. The elements are fabricated with a 3-axis CNC milling machine and the assembly code is printed on the corresponding element during the production process. As the connections are generated as notches it is necessary to take into account an opening angle required for an element when levering it into the structural framework.

\section{Pergola Hatt}

This pergola in the garden of the ETH guesthouse Villa Hatt is supported by five pre-existing columns (Fig. 19). One precondition was the use of hardwood beams with a given section and a maximum length of $2.50 \mathrm{~m}$. The heterogeneous structure 
of hardwood limits the use of longer solid wood beams. Therefore, the use of this kind of wood for reciprocal structures has promising potential.

During the design process the asymmetrical support positions result in beam lengths longer than the allowed maximum length (Fig. 20). Thus, the design is split in two intertwining different reciprocal structures: an inner wooden lattice and a surrounding frame on the principle of the 'Rainbow bridge' in Shandong (Di Carlo 2008) and the bridge sketched by Leonardo da Vinci in the Codex Atlanticus.

As a manufacturing constraint, each joint should be composed of a cross-lap and birth's mouth joint. Another pre-condition concerns the definition and automatic recognition of the joint geometry in the post-processing: the angle and the eccentricity of a pair of interlocking beams should be such that the intersection lines give four parallel lines. This is the case in a flat-vaulted form and/or the use of low

Fig. 19 Pergola Villa Hatt. Photo: courtesy Björn Siegrist
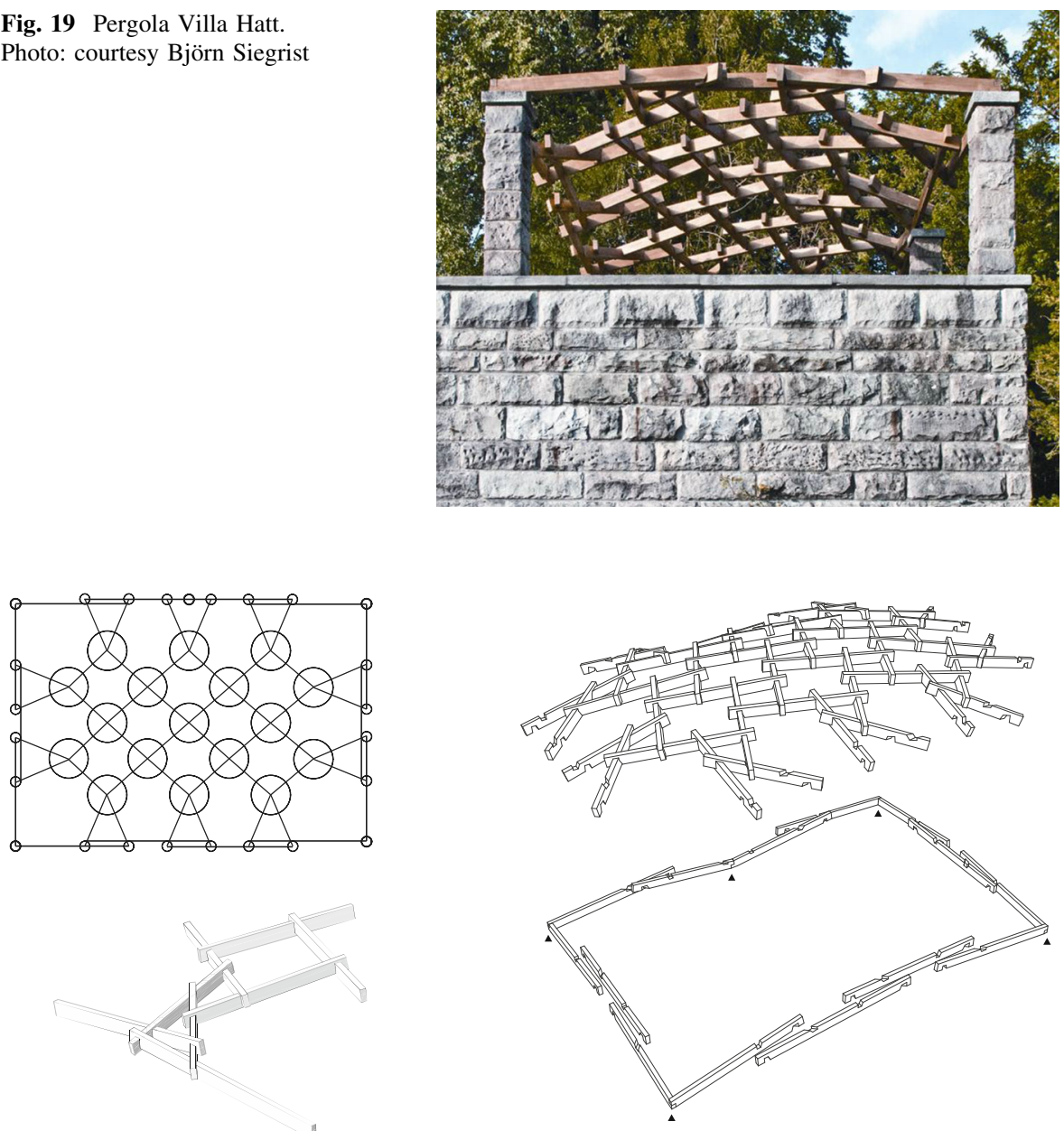

Fig. 20 Design of Hatt pergola, two intertwining reciprocal structures 


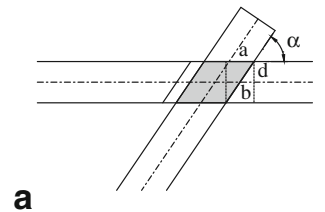

a

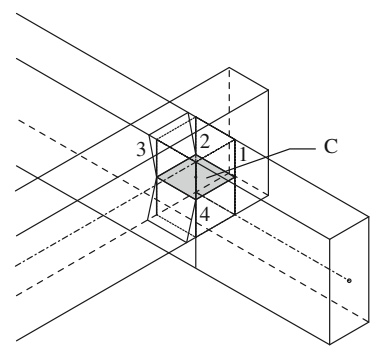

b
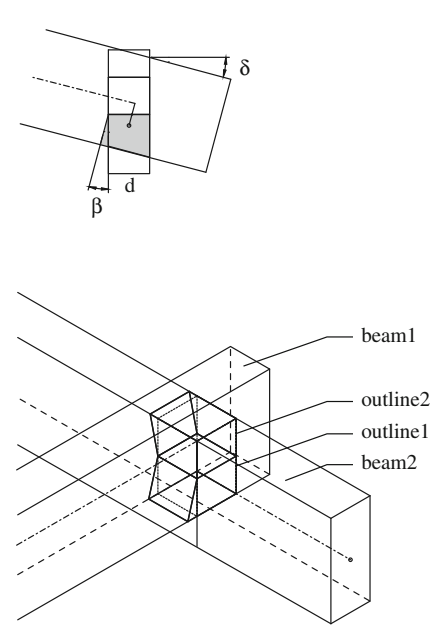

C

Fig. 21 a Joint geometry, b shared surface $C$, c joint outlines

eccentricity values. The parallel lines serve as surface normal to a shared surface $C$. By moving the surface along the parallel lines the depth of joint in each element can be adjusted.

The angle $\beta$ describes the opening of the notch to give a tolerance required for an element during the assembly (Fig. 21). This angle has been defined globally for the structure and is determined by means of physical models. The outline of the joint geometry and the beam geometry are then exported to CAD/CAM software for timber manufacturing, such as cadwork.

After the post-processing for the fabrication all pre-defined joints are executed by a joinery machine.

The transition from one structure to the other requires an assembly sequence of the elements levered into the framework beginning with the surrounding frame at the longest span.

\section{Conclusions}

The form-finding instrument described in this paper makes the family of reciprocal structures more accessible to the designer and makes a variety of architectural applications possible. Miscellaneous planar cells serve as initial configuration, subsequently the individual arrangement of these cells and the definition of different cell types allow the designer to choose the position and form of the boundaries and the scale of openings within the lattice framework. In an iterative process the elements are ordered spatially until the optimal configuration with the given parameters is obtained. This form-finding tool increases the design scope and the 
application potential of reciprocal systems and also supplies the data communication for the fabrication of the elements on digitally controlled production machines (CNC).

However, it does not supply a finished 'product' based on different input parameters. Rather, it assists the user in controlling the complex interdependencies of form and assembly during the design, and approximates step by step a suitable construction in terms of design and structural behaviour.

Hence, integrating the structural analysis and optimization is a challenge that is yet to be tackled. In planned further developments of the tool strategies of structural optimization therefore will play an important role. Different member cross sections will respond to different load situations in the structure. Moreover, different engagement lengths along an element should be actuated locally. In the postprocessing for manufacturing the definition of the joint geometry still has to be automated, so that several joint types can be recognized and computed.

This further development will allow a comparative survey of the reciprocal structures with other timber structures concerning material consumption, joint techniques and processing times.

Acknowledgments This work was mostly carried out within the framework of a research project in the Chair of Architecture and Construction, Professor Annette Spiro, at ETH Zurich by myself and the architect and researcher Nik Werenfels. The case studies were executed in a research-oriented teaching project supported by the Innovedum education fonds at ETH Zurich (Thönnissen and Werenfels 2011). Photographs by Peter Hauser, Christopher Rofe and Björn Sigrist reproduced by permission. All other illustrations by the author.

\section{References}

Baverel, Olivier. 2000. Nexorades: a family of interwoven space structures. Ph.D. thesis, University of Surrey.

Baverel, Olivier, Hoshyar Nooshin, and Yoshihiko Kuroiwa. 2004. Configuration processing of nexorades using genetic algorithms. Journal of the International Association for Shell and Spatial Structures 45(142): 99-108.

Bertin, Vito. 2002. Hebelstabwerke. Arch+ 159-160: 150-153.

Brown, Graham. 1989. Three-dimensional structures. United Kingdom Patent Office, Patent No. $2235479 B$.

Delaunay, Boris N. 1934. Sur la sphère vide. Bulletin of Academy of Sciences of the USSR 7(6): 793-800.

Di Carlo, Biagio. 2008. The wooden roofs of Leonardo and new structural research. Nexus Network Journal 10(1): 27-38.

Douthe, Cyril, and Olivier Baverel. 2009. Design of nexorades or reciprocal frame systems with the dynamic relaxation method. Computers and Structures 87: 1296-1307.

Houlsby G.T. 2014. John Wallis and the numberical analysis of structures. Nexus Network Journal 16, 1 in this same issue.

Kidokoro, R., and K. Goto. 2011. Rokko observatory-application of geometric engineering. In Proceedings of the International Symposium on Algorithmic Design for Architecture and Urban Design, Algode Tokyo 2011 (14-16 March 2011, Tokyo, Japan).

Parigi, Dario, Kirkegaard Poul H, and Sassone Mario. 2012. Hybrid optimization in the design of reciprocal structures. In IASS-APCS 2012 Proceedings: from spatial structures to spaces structures. The International Association for Shell and Spacial Structures.

Popovic Larsen, Olga. 1996. Reciprocal Frame Structures. Ph.D. thesis, University of Nottingham.

Popovic Larsen, Olga. 2008. Reciprocal frame architecture. Amsterdam: Elsevier. 
Popovic Larsen, Olga. 2009. Reciprocal frame Architecture in Japan. Pp. 1866-1876 in IASS Symposium 2009: Evolution and trends in design, analysis and construction of shell and spatial structures, eds. A. Domingo, and C. Lázaro, Valencia: Editorial de la UPV.

Pugnale, Alberto, and Sassone Mario. 2014. Structural reciprocity: critical overview and promising research/design issues. Nexus Network Journal 16, 1 in this same issue.

Rizzuto, J.P. 2007. Rotated mutually supported elements in truncated icosahedric domes. Journal of the International Association for Shell and Spatial Structures 48(1): 3-17.

Thönnissen, Udo, and Nik Werenfels. 2011. Reciprocal frames-teaching experiences. International Journal of Space Structures 26(4): 369-372.

Udo Thönnissen is a practising architect based in Zurich, Switzerland. He received his degree in architectural studies in 2001 from the Technical University of Aachen, Germany. Since 2007, he has been teaching as a lecturer at the Chair of Architecture and Construction, Professor Annette Spiro, in the Department of Architecture at ETH Zurich. Since 2010, he is leading a research project on reciprocal structures. He has been invited to lectures, critics and workshops at the architectural faculties of Aachen, Alghero, Barcelona, Cinicinnati, Liechtenstein, Mexico City and São Paulo. He is author of publications on architecture and construction in several books and journals. 